\title{
Síndrome de aorta media en pediatría
}

\section{Middle aortic syndrome in pediatric patients}

Pág. 48,53

Recibido: 20-04-2020

Aceptado: 07-05-2020

Dra. Karla Mora Membreño. Investigadora Independiente, Heredia, Costa Rica

Dra. Nicole D'Alaimo Brenes. Área de Salud Oreamuno Pacayas Tierra Blanca CCSS, Médico general, Costa Rica

Dra. Daniela D’Avanzo Zúñiga. Hospital Maximiliano Peralta CCSS,Médico general, Cartago, Costa Rica

\section{Resumen}

El síndrome de aorta media es una enfermedad vascular que afecta a la población pediátrica, también se le conoce como coartación abdominal, ya que este trastorno se presenta como estenosis de la aorta abdominal. Es común que exista compromiso extra aórtico, generalmente las arterias afectadas son las renales y mesentéricas. El paciente pediátrico con frecuencia exhibe hipertensión arterial severa, para la cual el tratamiento médico a menudo es insuficiente, por lo que muchos pacientes, para evitar las futuras complicaciones asociadas a la hipertensión arterial, terminan por requerir de alguna intervención endovascular o quirúrgica. Sin embargo, a pesar de un adecuado abordaje, la hipertensión arterial residual es frecuente, precisando tratamiento médico a largo plazo u otra intervención invasiva.

Palabras clave: Aorta; hipertensión; coartación aórtica; arteritis.

\section{Abstract:}

The middle aortic síndrome is a vascular disease affecting the pediatric population, it is also known as abdominal coarctation, since this disorder presents as stenosis of the abdominal aortic. It is common for extra aortic involvement to be present, and often the affected arteries are the renal and mesenteric. The pediatric patient frequently has severe arterial hypertension, for which medical treatment is often insufficient, for this reason, in order to avoid future complications associated with arterial hypertension, so many patients end up needing some endovascular or surgical approach. Howewer, despite an adequate management, residual arterial hypertension is frequent, requiring long-term medical treatment or other invasive intervention.

Key words: Aorta; hypertension; aortic coarctation; arteritis.

\section{Introducción:}

El síndrome de aorta media (SAM) es una rara enfermedad vascular que se presenta de manera predominante en el paciente pediátrico, con frecuencia esta enfermedad no se diagnostica de manera precoz, con el consecuente desarrollo de hipertensión arterial (HTA) y las complicaciones asociadas. La estrechez aórtica fue explicada por primera vez en el año 1835 por Schlessinger, sin embargo, el término de SAM fue acuñado hasta 1963 por Sen et al. Esta patología se caracteriza por estenosis de la aorta abdominal, sin embargo, puede asociar compromiso de la aorta torácica. Es frecuente la asociación de estenosis de las arterias viscerales, la arteria renal presenta estrechez en la mayoría de lo casos. El abordaje va dirigido al tratamiento oportuno de la enfermedad, para prevenir las complicaciones asociadas con la HTA, que con frecuencia es severa (1-4). El objetivo de este artículo de revisión bibliográfica

\section{(9) $(\mathbb{1 0} \Theta \Theta$}


es brindar una recopilación de la información más actualizada sobre el síndrome de aorta media en pediatría, con el fin de que el médico tratante pueda tener la sospecha clínica sobre esta enfermedad, para así disminuir el infradiagnóstico y las complicaciones asociadas con el abordaje tardío de la enfermedad.

\section{Método:}

Para la elaboración del presente artículo de revisión bibliográfica, la búsqueda de la información se realizó en las siguientes bases de datos: PubMed, Medline, UpToDate, The Journal of Thoracic and Cardiovascular Surgery (JTCVS), Elsevier y The Cochrane Library Plus. Se emplearon como términos de búsqueda las palabras claves: "middle aortic syndrome", "coartación aórtica", "hipertensión arterial en pediatria", "manejo del síndrome de aorta media". Todas las referencias son de relevancia médica científica, en idioma inglés y español. No se incluyó en la revisión las publicaciones sin respaldo por sociedades científicas, ni aquellas con una antigüedad mayor a 5 años. Se revisaron en total 34 bibliografías, entre artículos de revisión bibliográfica, reportes de casos, artículos de investigación, de los cuales se utilizaron un total de 16 artículos originales para la realización de este.

\section{Epidemiología:}

El SAM representa menos del $0,5 \%$ a $2 \%$ de todos los casos de estenosis aórtica $(5,6)$. Esta enfermedad afecta a la población pediátrica y adultos jóvenes, se considera una entidad potencialmente mortal debido a la HTA severa que estos pacientes podrían asociar (6). No existe diferencia sobre la incidencia de acuerdo con el género (3). La morbilidad y mortalidad son comunes en el SAM grave, la hipertensión renovascular es la complicación encontrada en mayor frecuencia (3). Sin tratamiento la tasa de supervivencia después de los 40 años es menor al $20 \%$, con complicaciones asociadas como infarto agudo del miocardio, falla cardíaca, hemorragia intracraneal, ruptura aórtica o falla renal $(3,7)$.

\section{Etiología:}

En aproximadamente un $64 \%$ de los casos del SAM no se realiza un diagnóstico subyacente, siendo así el origen idiopático la etiología de mayor prevalencia. Se clasifica como congénito cuando la enfermedad está presente desde el nacimiento $(2,3)$. EI SAM congénito se debe a un defecto embriológico, causado por el fracaso en la fusión normal de la aorta dorsal embrionaria, emparejada durante la cuarta semana de gestación, sin embargo, existe poca evidencia que lo demuestre (2-4).

EI SAM puede tener causas genéticas, tales como: neurofibromatosis tipo 1, mucopolisacaridosis, síndrome de Alagille y de Williams. También puede ser causado por enfermedades inflamatorias adquiridas, como la arteritis de Takayasu o por infecciones intrauterinas, en particular la rubéola $(2,3)$.

La arteritis de Takayasu es una vasculitis inmunomediada que afecta la aorta, las ramas principales de la aorta y las arterias pulmonares. Existe mayor prevalencia en el sexo femenino, con una distribución mayoritaria en el este de Asia (8).

\section{Patogénesis}

EI SAM se caracteriza por estenosis de la aorta abdominal, con frecuencia asocia compromiso de las ramas renales y esplácnicas, sin embargo, la patogénesis del SAM idiopático continúa siendo desconocida $(3,5)$. Para clasificar este trastorno se utiliza el punto anatómico de estenosis que se encuentra en la posición más cefálica (3).

El sitio más común para el SAM es el suprarrenal (29\%-60\%), el infrarrenal se encuentra con menor frecuencia $(8 \%-15 \%)$, no obstante, la ubicación puede variar según la etiología subyacente. Los casos causados por trastornos genéticos están mayormente asociados con la estenosis suprarrenal $(2,3)$. La localización de la lesión aórtica en el SAM idiopático es intrarrenal en un $19 \%-52 \%$ de los casos, suprarrenal en un $11 \%$ $40 \%$, infrarrenal un $19 \%-25 \%$ y difusa un $12 \%$ de 
los casos (5). La tasa de aneurismas midaórticos asociados al SAM es aproximadamente del $11 \%$, la mayoría de los casos reportados son causados por enfermedades inflamatorias $(2,3)$.

Alrededor del $70 \%-85 \%$ de los pacientes pediátricos presentan compromiso extra aórtico, siendo la mayoría de los casos de etiología genética. La afectación de la arteria renal tiene una incidencia del $70 \%-80 \%$, de los cuales, $60 \%$ presenta estenosis bilateral de esta arteria y $20 \%$ estenosis unilateral (2-4). Los segundos vasos involucrados con mayor frecuencia son los mesentéricos, la arteria mesentérica superior se afecta un $25 \%-30 \%$ de los casos, el tronco celiaco un $22 \%$ y la arteria mesentérica inferior rara vez se ve comprometida $(3,4,9)$. Los vasos retinianos y cerebrales son lesionados en menor medida, con tasas menores al $10 \%(2,3)$. Los casos debidos a arteritis evolucionan en su mayoría como una enfermedad aórtica aislada (3).

Por otro lado, en la enfermedad de Takayasu se han encontrado casos con afectación de la arteria subclavia izquierda (6). EI SAM debido a esta arteritis afecta generalmente la porción proximal de las ramas principales de la aorta. Además, el principal contribuyente a la morbimortalidad asociado con la enfermedad de Takayasu es la falla cardíaca (10).

\section{Presentación clínica}

EI SAM con frecuencia se presenta antes de los 18 años, aquellos pacientes que debutan antes del primer año tendrán una enfermedad de mayor gravedad. Lo anterior se debe a que estos pacientes presentan mayor cantidad de ramas arteriales con estenosis de alto grado, en lugar de solamente presentar estenosis midaórtica. Gracias a los avances de las herramientas radiológicas por imágenes, la edad media al momento del diagnóstico ha disminuido a los 7 años de edad $(1,3)$.

- Hipertensión arterial: En la población pediátrica la HTA tiene una incidencia del $1 \%-2 \%$, alrededor de $2 / 3$ de los casos son de etiología secundaria, la enfermedad renal-parenquimatosa y renovascular son las más comunes. La estenosis de la aorta suprarrenal y/o de las arterias renales inducen hipoperfusión renal, la cual activa el sistema renina angiotensina aldosterona, con la consecuente HTA renovascular. La HTA en pediatría se define como una presión arterial sobre el percentil 95 para la edad, sexo y altura, o una medición mayor a 130/80 en niños mayores de 13 años. El paciente pediátrico con el SAM generalmente presenta HTA severa, la cual en ocasiones se descubre como un hallazgo incidental durante el estudio de la enfermedad $(2,7-9,11,12)$.

Los pacientes con SAM severo pueden cursar con HTA refractaria, la cual, de no ser tratada, conlleva a complicaciones tales como enfermedad coronaria, insuficiencia cardíaca congestiva, hipertrofia del ventrículo izquierdo ( $\mathrm{HVI})$ o evento cerebrovascular (2,3). La HVI sirve como marcador para el daño a órgano final causado por la HTA severa, se ha visto que, tras el manejo adecuado de la HTA la hipertrofia podría llegar a resolver. Además, los pacientes con enfermedad severa que asocian compromiso de los vasos renales de alto grado podrían presentar enfermedad renal en etapa terminal (3).

- Examen físico: Los hallazgos clínicos varían según el grado de estenosis vascular, su ubicación, así como de la enfermedad asociada del paciente. Se debe realizar medición de la presión arterial en las cuatro extremidades, encontrándose discrepancia en la medición entre miembros superiores e inferiores. La evaluación de los pulsos periféricos es parte importante de la evaluación clínica, la mayoría de los pacientes manifiestan ausencia de los pulsos femorales. Es frecuente la auscultación de un soplo abdominal. Es posible encontrar hallazgos compatibles con claudicación de las extremidades inferiores, así como de angina intestinal, ambos son consecuencia de un flujo sanguíneo disminuido hacia la parte inferior del cuerpo, debido a una estenosis significativa de la aorta abdominal $(2,3,7,8)$.

\section{Diagnóstico}


El ultrasonido doppler es la prueba inicial que se realiza en el paciente pediátrico con sospecha de hipertensión renovascular. Los estudios por imágenes no invasivos como la angiografía tomográfica computarizada y la angiografía por resonancia magnética proporcionan información anatómica detallada de las estructuras vasculares, no obstante, en niños pequeños su valor es limitado. Por el riesgo conocido de insuficiencia renal en estos pacientes, se debe de tener en cuenta la función renal cuando se utiliza el gadolinio durante la resonancia magnética. La angiografía transcatéter es el método de elección cuando los estudios por imágenes no invasivos no son concluyentes, esta prueba es el estándar de oro para el diagnóstico del SAM, además de realizar el diagnóstico, también permite la realización de intervenciones durante el estudio $(3,7,11)$.

El flujo sanguíneo arterial del riñón se puede afectar en el SAM severo, provocando elevación de los niveles de renina, creatinina y urea en plasma. Las alteraciones en otros estudios de laboratorio dependen de la etiología subyacente, como en la arteritis de Takayasu, en la cual hay presencia de anemia, elevación de la velocidad de eritrosedimentación y de la proteína $\mathrm{C}$ reactiva (3).

\section{Tratamiento}

Existen tres modalidades terapéuticas para el abordaje del SAM, las cuales son el tratamiento farmacológico, endovascular y quirúrgico. El tratamiento inicial va dirigido a controlar la presión arterial, para prevenir las complicaciones a largo plazo relacionadas con la HTA $(1,2,7,13)$. El objetivo principal de la intervención quirúrgica es restaurar el flujo sanguíneo en la aorta distal y sus ramas. Además, la revascularización de la aorta estenótica es esencial para aliviar los síntomas y aumentar la sobrevida a largo plazo. Sin embargo, no se han desarrollado guías estandarizadas con las indicaciones para el abordaje invasivo del SAM, no obstante, la HTA refractaria, la insuficiencia renal y cardiomiopatía son desencadenantes habituales para la intervención endovascular o quirúrgico $(2,3,13,14)$.
Todo paciente sometido a un procedimiento de revascularización, así como aquellos con alto riesgo para la formación de trombos, deben recibir dosis bajas de aspirina o terapia antiplaquetaria dual para la prevención primaria y secundaria (12).

- Tratamiento farmacológico: Existen diversos agentes anti hipertensivos para el manejo de la HTA, sin embargo, los fármacos recomendados para el control inicial son los bloqueadores de canales de calcio y/o beta bloqueadores. En el caso de HTA no controlada con alta actividad del sistema renina-angiotensina-aldosterona (SRAA) o hipertensión renovascular, los fármacos de preferencia son los inhibidores de la enzima convertidora de angiotensina y antagonistas del receptor de angiotensina II; los diuréticos tiazida se administran en el paciente con insuficiencia cardíaca. Por lo tanto, la elección del fármaco anti hipertensivo debe individualizarse según las características de cada paciente $(2,3,12)$.

Los casos idiopáticos tienen altas tasas de HTA refractaria a pesar del tratamiento médico, en comparación con el SAM de otras etiologías. En los pacientes con estenosis aórtica leve a moderada, el tratamiento farmacológico con anti hipertensivos orales puede ser satisfactorio, por otro lado, a menudo la HTA en el SAM grave no responde al manejo médico por si solo (1-4).

Existe debate con respecto al manejo de la HTA causada por arteritis, ya que en varios estudios clínicos se ha preferido el abordaje farmacológico de la HTA refractaria antes que el quirúrgico, por otra parte, hay quienes prefieren retrasar las intervenciones invasivas en el paciente pediátrico hasta que se haya completado el crecimiento somático, siempre y cuando no exista HTA refractaria concomitante $(2,3)$.

- Tratamiento endovascular: Los procedimientos endovasculares se realizan con menor frecuencia en comparación con los quirúrgicos. Las dos técnicas mayormente utilizadas son la dilatación con balón y la endoprótesis vascular $(2,9,15)$. La tasa de reintervención para el abordaje endovascular a los 5 años de seguimiento alcanza hasta un $67 \%$, el factor más común es la 
reestenosis en la endoprótesis vascular. Entre las complicaciones asociadas con este procedimiento se encuentran: trombosis, sangrado, evento cerebrovascular y reestenosis; las cuales se asocian con mayor frecuencia a pacientes con biomarcadores inflamatorios elevados, sugiriendo que un mayor grado de inflamación podría ser un factor de riesgo para futuras complicaciones $(2,3)$. A nivel de las arterias renales se puede realizar la colocación de una endoprótesis vascular, sin dejar de lado el alto riesgo de oclusión de las arterias viscerales tras la colocación de una endoprótesis vascular en la aorta abdominal $(3,9)$.

- Tratamiento quirúrgico: La cirugía abierta sigue siendo el procedimiento que con mayor frecuencia se realiza, a esta intervención se someten aproximadamente un $40 \%-55 \%$ de todos los pacientes que requieren de un abordaje invasivo $(2,3,9)$.

Existen diversas técnicas quirúrgicas, y su elección depende de factores como, la ubicación de la estenosis, la longitud del segmento afectado, así como el compromiso de vasos viscerales $(2,3)$. Dentro de los métodos quirúrgicos se encuentran la derivación aortoaórtica, la angioplastía utilizando injerto protésico y la reparación aórtica primaria posterior al alargamiento aórtico (1-3,9,13,15). A pesar de la posible efectividad en el uso de material protésico, en el paciente pediátrico se evita en la medida de lo posible, ya que podría requerir procedimientos adicionales debido al crecimiento somático. Sin embargo, la tasa de ausencia de reintervención a los 10 años de seguimiento es de $72 \%$. Las complicaciones que con mayor frecuencia se asocian con la cirugía abierta son, la trombosis, infección y aneurisma anastomótico $(1,3,9,13)$.

En los últimos años se han desarrollado dos nuevas técnicas quirúrgicas que evitan el uso de injertos protésicos. La primera de ellas consiste en la utilización de un expansor de tejido, que se encarga de alargar la aorta distal no estenótica; se realiza la resección completa de la aorta estenosada para finalizar con una anastomosis primaria, este procedimiento se prefiere para casos con estenosis de segmento corto. La segunda técnica consiste en utilizar la arteria mesentérica como injerto, ya que tiene significativo potencial de crecimiento, se realiza en pacientes con estenosis de segmento largo (3).

En pacientes con SAM por arteritis de Takayasu la cirugía abierta se considera el estándar de oro para el abordaje, sin embargo, diversos estudios clínicos han utilizado la colocación de una endoprótesis vascular como una estrategia efectiva y segura $(8,14)$.

Es importante la monitorización regular de la glucosa en sangre en el paciente neonato al que se le practique cirugía abierta, así como mantener la normoglicemia, ya que estos pacientes tienen mayor riesgo de presentar hipoglicemia. Los factores de riesgo para el desarrollo de hipoglicemia a tomar en cuenta son, la prematuridad, el estrés perinatal, pequeño para la edad gestacional y la diabetes materna 16.

\section{Conclusión}

Durante la evaluación clínica del paciente pediátrico no es habitual la medición de la presión arterial, por esta razón muchos pacientes no son diagnosticados en etapas precoces de la enfermedad y presentan riesgo elevado de complicaciones asociadas con la enfermedad hipertensiva. Es por esto, que la sospecha clínica y el examen físico preciso, incluyendo la medición de la presión arterial en las cuatro extremidades y la evaluación de los pulsos periféricos son herramientas clave para poder realizar el diagnóstico precoz del SAM, para así prevenir las complicaciones asociadas con la HTA. Sin embargo, a pesar de un diagnóstico oportuno y adecuado abordaje tanto médico como quirúrgico, es frecuente la presencia de HTA residual que requiera el uso de anti hipertensivos y en algunos casos de intervención invasiva, además del monitoreo a largo plazo.

Según la bibliografía revisada, las áreas débiles del tema son las indicaciones para el abordaje

\section{(ㄱ) $(1) \Theta \theta$}


invasivo del paciente con el SAM, ya que no existen guías estandarizadas con las indicaciones para el abordaje quirúrgico y endovascular, lo cual aumenta la complejidad del abordaje y supone un reto al médico tratante. Por otra parte, todos los estudios revisados comparten la importancia del diagnóstico temprano, con el apoyo de la evaluación clínica del paciente y los estudios por imágenes, ya que el pronóstico de la enfermedad está influenciado por la aparición de complicaciones hipertensivas.

\section{Referencias bibliográficas}

(1). Kim H, Lee E, Vakili K, Stein D, Ferguson M, Porras D et al. Mesenteric Artery Growth Improves Circulation (MAGIC) in Midaortic Syndrome. Ann Surg [Internet]. 2018 [Consultado 14 Enero 2020]; 267(6):e109-e111. Disponible en: https://doi. org/10.1097/sla.0000000000002540

(2). Rumman R, Nickel C, Matsuda-Abedini M, Lorenzo A, Langlois V, Radhakrishnan S et al. Disease Beyond the Arch: ASystematic Review of Middle Aortic Syndrome in Childhood. Am J Hypertens [Internet]. 2015 [Consultado 10 Enero 2020]; 28(7):833-841. Disponible en: https://doi.org/10.1093/ajh/ hpu296

(3). Forman N, Sinskey J, Shalabi A. A Review of Middle Aortic Syndromes in Pediatric Patients. J Cardiothorac Vasc Anesth [Internet]. 2019 [Consultado 10 Enero 2020]; 0(0)1-7. Disponible en: http://doi.org/10.1053/j.jvca.2019.07.130

(4). Ahn K, Yoon J, Kim G, Kwon B, Bae E, Noh C. Idiopathic midaortic syndrome with malignant hypertension in 3-yearold boy. Korean J Pediatr [Internet]. 2016 [Consultado 12 Enero 2020]; 59(Suppl 1):S84-S87. Disponible en: http://doi. org/10.3345/kjp.2016.59.11.s84

(5). MirA, Stam B, Sperrazza C.Arare cause of cardiomyopathy in an infant: Middle aortic syndrome. Cardiol Young [Internet]. 2017 [Consultado 14 Enero 2020]; 27(4):794-796. Disponible en: http://doi.org/10.1017/s1047951116002018

(6). Hakim K, Drissa M, Msaad H, Ouarda F. Contribution of imaging modalities in the diagnosis of middle aortic syndrome. Egypt Heart J [Internet]. 2018 [Consultado 14 Enero 2020]; 70(3):225-228. Disponible en: http://doi.org/10.1016/j. ehj.2018.06.001

(7). Yakut K, Erdogan I. Case report of a rarely seen long segment middle aortic syndrome. Turk Kardiyol Dern Ars [Internet]. 2017 [Consultado 12 Enero 2020]; 45(2):181-182. Disponible en: http://doi.org/10.5543/tkda.2016.64606

(8). Morrissey S, Tan K, Byrne J. Takayasu arteritis with middle aortic syndrome and mesenteric ischemia treated by aortic stenting. J Vasc Surg Cases Innov Tech [Internet]. 2017 [Consultado 10 Enero 2020]; 3(3):168-170. Disponible en: http://doi.org/10.1016/j.jvscit.2017.06.004

(9). Day E, Stojanovic J, Karunanthy N, Qureshi S, Reidy J, Sinha M. Middle aortic syndrome-an 8-year story of pills, pretty balloons and struts. Pediatr Nephrol [Internet]. 2015 [Consultado 11 Enero 2020]; 30:1361-1364. Disponible en: https://doi.org/10.1007/s00467-015-3118-2

(10). Fan L, Zhang H, Cai J, Ma W, Song L, Lou Y. Middle aortic syndrome because of pediatric Takayasu arteritis admitted as acute heart failure. J Hypertens [Internet]. 2018 [Consultado 10 Enero 2020]; 36(10):2118. Disponible en: http://doi.org/10.1097/HJH.0000000000001847

(11). Agrawal H, Moodie D, Qureshi A, Acosta A, Hernandez $\mathrm{J}$, Braun $\mathrm{M}$ et al. Interventions in children with renovascular hypertension: A 27-year retrospective single-center experience. Congenit Heart Dis [Internet]. 2018 [Consultado 13 Enero 2020]; 13(3):1-7. Disponible en: http://doi. org/10.1111/chd.12608

(12). Fan L, Zhang H, Cai J, Yang L, Wei D, Yu J et al. Clinical Course, Management, and Outcomes of Pediatric Takayasu Arteritis Initially Presenting With Hypertension: A 16-year overview. Am J Hypertens [Internet]. 2019 [Consultado 12 Enero 2020]; 32(10):1021-1028. Disponible en: https://doi. org/10.1093/ajh/hpz103

(13). Thompson J, Sprouse K, Mir A, Burkhart H. Successful staged management of neonatal midaortic syndrome. J Thorac Cardiovasc Surg [Internet]. 2018 [Consultado 14 Enero 2020]; 157(5):e281-e282. Disponible en: https://doi. org/10.1016/j.jtcvs.2018.11.067

(14). Che W, Xiong H, Jiang $X$, Dong $H$, Zou $Y$, Yang $Y$ et al. Stenting for middle aortic syndrome caused by Takayasu arteritis-immediate and long-term outcomes. Catheter Cardiovasc Interv [Internet]. 2018 [Consultado 10 Enero 2020]; 91(S1):623-630. Disponible en: http://doi.org/10.1002/ ccd. 27492

(15). Zartner P, Hart C, Schneider M. Severe midaortic syndrome: a stepwise approach to treatment with drug-eluting balloons: a case report. Eur Heart J Case Rep [Internet]. 2019 [Consultado 13 Enero 2020]; 3(1):1-3. Disponible en: http://doi.org/10.1093/ehjcr/ytz017

(16). Arumainathan R, Stendall C, Visram A. Management of fluids in neonatal surgery. BJA Educ [Internet]. 2018 [Consultado 11 Enero 2020]; 18(7):199-202. Disponible en: https://doi.org/10.1016/j.bjae.2018.03.006 